\title{
A bounded dynamic programming algorithm for the MMSP-W considering workstation dependencies and unrestricted interruption of the operations
}

\author{
Joaquín Bautista, Alberto Cano, Rocío Alfaro \\ Nissan Chair ETSEIB - UPC, Barcelona \\ Escola Tècnica Superior d'Enginyeria de Barcelona \\ Universitat Politècnica de Catalunya \\ Barcelona, Spain \\ joaquin.bautista@upc.edu, alberto.cano-perez@upc.edu, rocio.alfaro@upc.edu
}

\begin{abstract}
In this paper, we propose a procedure based on Bounded Dynamic Programming $(B D P)$ to solve the MixedModel Sequencing Problem with Workload Minimisation (MMSP-W), with serial workstations and unrestricted (or free) interruption of the operations. We performed a computational experiment with 225 instances from the literature. The results of our proposal are compared with those obtained through the CPLEX solver.
\end{abstract}

Scheduling; sequencing; work overload; Dynamic Programming, linear programming

\section{INTRODUCTION}

In mixed-model manufacturing lines, which are common in Just-in-time $(J I T)$ and Douki Seisan $(D S)$ ideologies, several variants of one or more products can be handled. This flexibility determines the order in which the units are treated to drastically reduce intermediate stocks and to capitalise on the time available for manufacturing.

In these settings, we can choose two basic categories of objectives [1].

A. Work overload or lost work. The greatest possible reduction of work overload that may appear due to production programs with mixed products.

B. JIT. The greatest possible reduction of the levels of stocks in the system.

For category A, in addition to a relative focus on maximising the total amount of work completed [2], the excess effort that must be applied over time for certain operations can be modulated [3].

Using this perspective, sequencing problems can be grouped into three categories: (1) Mixed-model sequencing; (2) Car sequencing; and (3) Level scheduling.

Within this framework, [1] provided an up-to-date review of the literature.

The present study can be placed in category A.1., and it takes the minimisation of the total work overload as its optimisation criteria.

Overload, or excess effort, is a measurement, in units of time, of work that cannot be completed at the standard rhythm of an established activity, within the time granted to the workstations (cycle). This overload may arise when the processing time of a unit at a workstation is greater than the cycle time [2], although there may be a certain amount of play associated with extended cycles, which is called the length of the workstation or the time window.

When faced with a foreseeable workstation overload, at least three types of measures can be taken:

I. Stop the line and complete the pending work using reinforcements $[4,5]$.

II. Let the unit pass and finish the pending work in a final line at a later time. $[2,6,7,8]$.

III. Increase productive activity above the standard, using the assistance of reinforcement operators or previously programmed robotised systems.

The present study considered measures in categories II and III for handling work overloads.

Mixed-Model Sequencing Problem with Workload Minimisation $(M M S P-W)$ is an NP-hard problem [2], for which several alternative solutions have been proposed. These include exact procedures based on branch and bound [9], dynamic programming [2, 10], heuristic procedures based on local search $[6,11]$, greedy algorithms with priority rules $[6,12]$, meta-heuristics $[13,14]$ and beam search [15].

For this paper, we used a procedure based on Bounded Dynamic Programming $(B D P)$. This procedure combines features of dynamic programming with features of branch and bound algorithms. The principles of $B D P$ have been described by [16] and [17]. Previous work on similar approaches has been done by [10] and [18]. This procedure is computationally competitive with the integer linear programming. Our proposal contains the following:

1. A model for the $M M S P-W$ that combines properties from Yano and Scholl models.

2. A procedure based on dynamic programming to solve this problem.

3. A computational experiment with reference instances from the literature to compare the results offered by $B D P$ with those offered by integer linear programming (CPLEX solver).

This paper is organised as follows. Section II presents a model for the $M M S P-W$ with serial workstations and with unrestricted interruption of the operations. Section III shows an illustrative example. Section IV describes the basic elements and the application of the proposed $B D P$ procedure. Section $\mathrm{V}$ describes the computational experiments with reference instances from the literature. Finally, Section VI shows the conclusions of the study. 


\section{MODEL FOR MMSP-W WITH SERIAL WORKSTATIONS AND UNRESTRICTED INTERRUPTION OF THE OPERATIONS}

For the $M M S P-W$ with serial workstations, unrestricted interruption of the operations and work overload minimisation, we take as reference the model $M 4$, proposed by [10].

The extended model $M 4^{\prime}$ is focused on minimising the total overload (i.e., maximising the total work performed) and uses relative start instants of the units and considers more than one homogeneous processor at each workstation. The parameters and variables of this model are presented below.

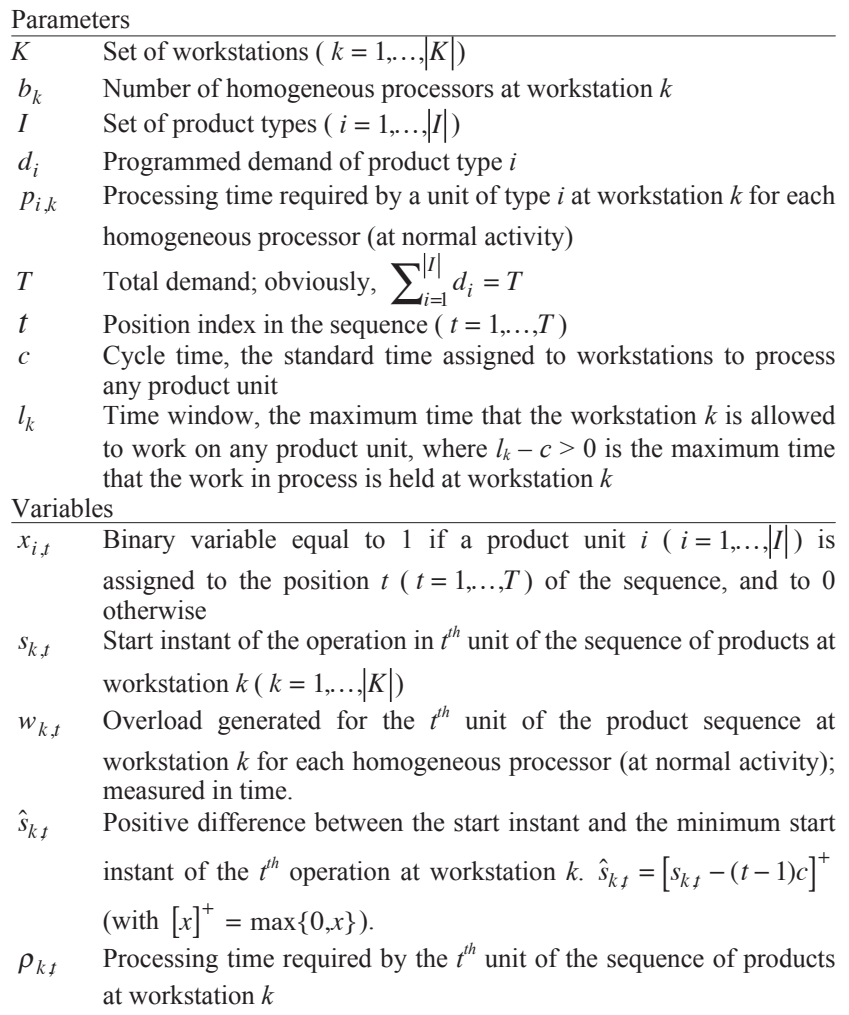

$c \quad$ Cycle time, the standard time assigned to workstations to process any product unit

$l_{k} \quad$ Time window, the maximum time that the workstation $k$ is allowed to work on any product unit, where $l_{k}-c>0$ is the maximum time that the work in process is held at workstation $k$

$x_{i, t} \quad$ Binary variable equal to 1 if a product unit $i(i=1, \ldots,|I|)$ is assigned to the position $t(t=1, \ldots, T)$ of the sequence, and to 0 otherwise

$s_{k, t} \quad$ Start instant of the operation in $t^{\text {th }}$ unit of the sequence of products at workstation $k(k=1, \ldots,|K|)$

$w_{k, t} \quad$ Overload generated for the $t^{\text {th }}$ unit of the product sequence at workstation $k$ for each homogeneous processor (at normal activity); measured in time.

$\hat{s}_{k f} \quad$ Positive difference between the start instant and the minimum start instant of the $t^{t h}$ operation at workstation $k . \hat{s}_{k f}=\left[s_{k f}-(t-1) c\right]^{+}$ (with $[x]^{+}=\max \{0, x\}$ ).

$\rho_{k f} \quad$ Processing time required by the $t^{\text {th }}$ unit of the sequence of products at workstation $k$

Model M4':

$$
\operatorname{Min} W=\sum_{k=1}^{|K|}\left(b_{k} \sum_{t=1}^{T} w_{k, t}\right)
$$

Subject to:

$$
\begin{aligned}
& \forall i=1, \ldots,|I| \\
& \forall t=1, \ldots, T \\
& \forall k=1, \ldots,|K| ; \forall t=1, \ldots, T \\
& \forall k=1, \ldots,|K| ; \forall t=1, \ldots, T
\end{aligned}
$$

$$
\begin{array}{ll}
\hat{s}_{k, t} \geq \hat{s}_{k, t-1}+\rho_{k t-1}-w_{k t-1}-c & \forall k=1, \ldots,|K| ; \forall t=2, \ldots, T \\
\hat{s}_{k, t} \geq \hat{s}_{k-1 t}+\rho_{k-1, t}-w_{k-1 \neq}-c & \forall k=2, \ldots,|K| ; \forall t=1, \ldots, T \\
\hat{s}_{k, t}+\rho_{k \neq}-w_{k t} \leq l_{k} & \forall k=1, \ldots,|K| ; \forall t=1, \ldots, T \\
\hat{s}_{k, t} \geq 0 & \forall k=1, \ldots,|K| ; \forall t=1, \ldots, T \\
w_{k, t} \geq 0 & \forall k=1, \ldots,|K| ; \forall t=1, \ldots, T \\
x_{i, t} \in\{0,1\} & \forall i=1, \ldots,|I| ; \forall t=1, \ldots, T
\end{array}
$$

In the model, the equivalent objective function (1) is represented by the total overload $(W)$. Constraint (2) requires that the programmed demand be satisfied. Constraint (3) indicates that only one product unit can be assigned to each position of the sequence. Constraint (4) links the processing times required by the types of product with the processing times required by the units in the sequence. Constraint (5) establishes upper bounds for the overload values through the required processing times by the units on the sequence. Constraints (6)-(9) constitute the set of relative start instants of the operations at each workstation and the processing times applied to the products. Constraint (10) indicates that the generated overloads are not negative. Constraint (11) requires the assigned variables to be binary. Finally, constraint (12) fixes the start of operations.

\section{AN ILLUSTRATIVE EXAMPLE}

To illustrate the model formulated above, we present the following example.

There are six units of product $(T=6)$, of which three are type $A$, one is type $B$ and two are type $C$, with a total work required $V_{0}=104$. The units are processed at three workstations $(|K|=3)$ with different numbers of processors $\left(b_{k}\right)$; the processing times for each processor (at normal activity) for each type of unit $i(A, B, C)$ at each workstation $k\left(m_{1}, m_{2}, m_{3}\right)$ are listed in Table I.

TABLE I. PROCESSING TIMES $\left(p_{i, k}\right)$, NUMBER OF HOMOGENEOUS PROCESSORS $\left(b_{k}\right)$ AND TOTAL WORK $\left(V_{0}\right)$ REQUIRED BY EACH TYPE OF UNIT AT EACH WORKSTATION

\begin{tabular}{|c|c|c|c|c|}
\hline & $\boldsymbol{A}\left(d_{A}=3\right)$ & $\boldsymbol{B}\left(d_{B}=1\right)$ & $\boldsymbol{C}\left(d_{C}=2\right)$ & $b_{k}$ \\
\hline$m_{1}$ & 5 & 4 & 3 & 1 \\
$m_{2}$ & 5 & 4 & 4 & 2 \\
$m_{3}$ & 4 & 3 & 5 & 1 \\
\hline Total & $19\left(V_{0}(A)=57\right)$ & $15\left(V_{0}(B)=15\right)$ & $16\left(V_{0}(C)=32\right)$ & $V_{0}=104$ \\
\hline
\end{tabular}

Furthermore, $c=4$ (cycle time) and $l_{k}=6$ for $k=1, \ldots, 3$ (length of the workstation or time window).

Fig. 1 shows a Gantt diagram of the optimal solution offered by model $M 4^{\prime}$. The sequence of products that presents the minimum total overload is $C-B-A-C-A-A$. The total work performed is $V=101$, and the overload, which is concentrated between workstations $m_{1}$ and $m_{2}$, is $W=3$ (the grey area in Fig.1). 


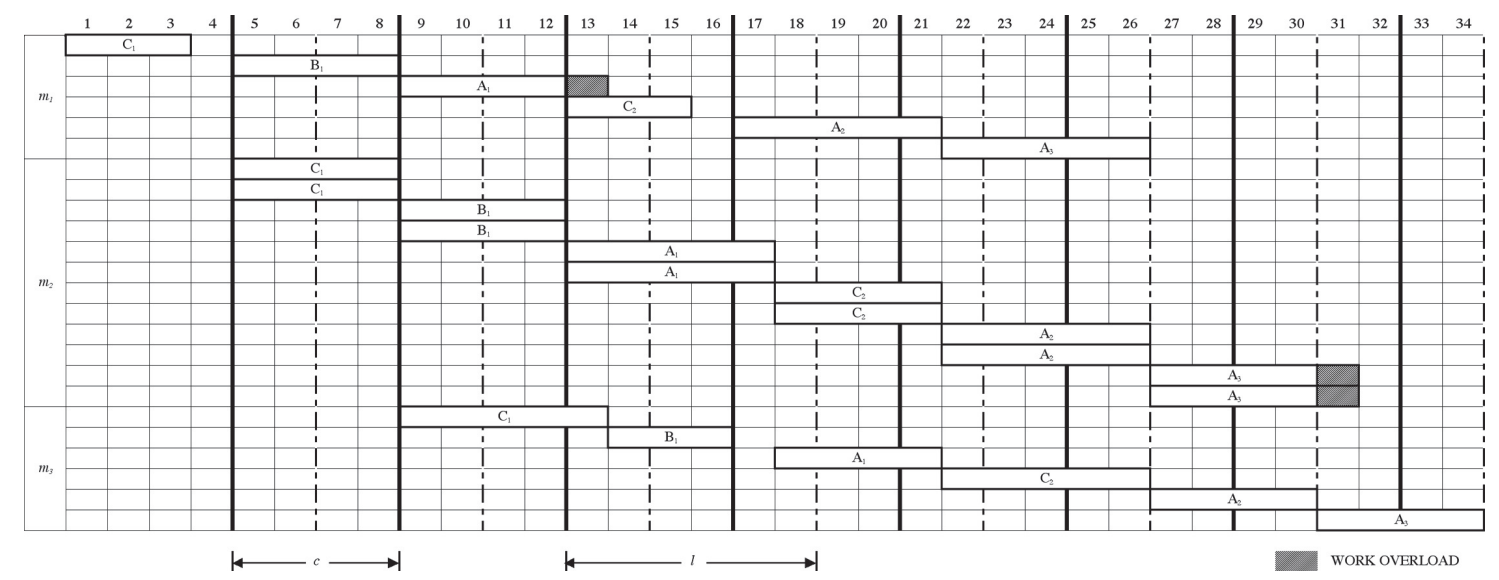

Figure 1. Gantt chart for the optimum solution for the example provided by $M 4$ '.

\section{BDP FOR THE MMSP-W}

This section presents the basic elements of the $B D P$ procedure applied to the resolution of $M M S P-W$ with serial workstations and unrestricted interruption of the operations.

\section{A. Global and partial Bounds}

Given a vertex of the stage $t$, reached through a partial sequence $\pi(t)=\left\{\pi_{1}, \pi_{2}, \ldots, \pi_{t}\right\}$, the overall bound for $W$ and a partial bound for the complement $R(\pi(t))$ associated to the sequence or segment $\pi(t)$ can be determined according to the schema presented in Fig. 2.

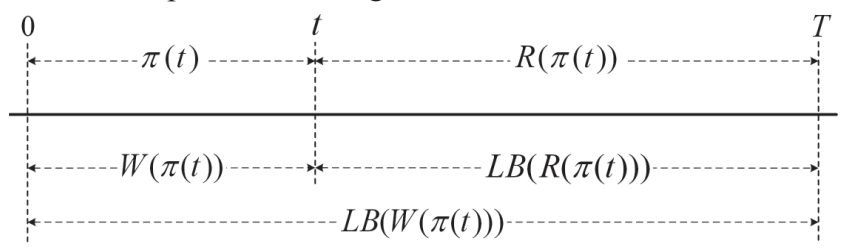

Figure 2. Bound scheme for a partial sequence $\pi(t)$

To obtain the bounds of the overloads associated to $\pi(t)$ and $R(\pi(t))$, we impose the following conditions to $M 4$ ':

- $\quad$ The values of the variables $x_{i, \tau}(i=1, \ldots,|I| ; \tau=1, \ldots, t)$ are fixed by $\pi(t)$ :

$$
x_{i, \tau}=\left\{\begin{array}{l}
1, \text { if } \pi_{\tau}=i \\
0, \text { if } \pi_{\tau} \neq i
\end{array}\right\} \Leftrightarrow\left\{\begin{array}{l}
x_{\pi_{\tau}, \tau}=1 \\
x_{i, \tau}=0, \text { if } i \neq \pi_{\tau}
\end{array}\right\}
$$

- The binary condition is relaxed for the variables $x_{i, \tau}(i=1, \ldots,|I| ; \tau=t+1, \ldots, T)$ :

$$
0 \leq x_{i, \tau} \leq 1 \quad i=1, \ldots,|I| ; \tau=t+1, \ldots, T
$$

The result is the following linear program $\left(L B \_M 4^{\prime}\right)$ :

$$
\operatorname{Min} L B(W(\pi(t)))=\sum_{k=1}^{|K|}\left(b_{k} \sum_{t=1}^{T} w_{k, t}\right)
$$

Subject to:

(2)-(10) and (12) from $M 4^{\prime}$

$x_{\pi_{\tau}, \tau}=1$

$$
\begin{aligned}
& \forall \tau=1, \ldots, t \\
& \forall i=1, \ldots,|I| ; \forall \tau=t+1, \ldots, T
\end{aligned}
$$

The previous linear program provides an overall bound of the total overload $(L B(W(\pi(t))))$, the value of the overload associated to the segment $\pi(t)(W(\pi(t)))$ and a bound of the overload associated to the complement $R(\pi(t))$ $(L B(R(\pi(t))))$. These values can be calculated as follows:

$$
\begin{aligned}
& W(\pi(t))=\sum_{k=1}^{|K|}\left(b_{k} \sum_{\tau=1}^{t} w_{k, \tau}\right) \\
& L B(R(\pi(t)))=\sum_{k=1}^{|K|}\left(b_{k} \sum_{\tau=t+1}^{T} w_{k, \tau}\right)
\end{aligned}
$$

The relative completion instants $\left(\hat{e}_{k, t}\right)$ of the last operation of the partial sequence $\pi(t)$, in each workstations, can be obtained from $L B \quad M 4$ ' as follows:

$$
\hat{e}_{k, t}=\hat{s}_{k, t}+\rho_{k, t}-w_{k, t} \quad \forall k=1, \ldots,|K|
$$

\section{$B$. Graph associated with the problem}

Similar to [10], we can build a linked graph without loops or direct cycles of $T+1$ stages. The set of vertices in level $t(t=0, \ldots, T)$ will be noted as $J(t) . J(t, j)(j=1, \ldots$, $|J(t)|)$ being a vertex of level $t$, which is defined by the tuple $(\vec{q}(t, j), \vec{e}(t, j), \pi(t, j), L B(W(\pi(t, j))))$, where:

- $\vec{q}(t, j)=\left(q_{1}(t, j), \ldots, q_{|I|}(t, j)\right)$ represents the vector of demand satisfied.

- $\vec{e}(t, j)=\left(e_{1}(t, j), \ldots, e_{|K|}(t, j)\right)$ is the vector of absolute completion instants of the operations at the workstations.

- $\pi(t, j)$ represents the sequence of $t$ units of product associated to the vertex.

- $L B(W(\pi(t, j)))$ is the bound of the overall overload, considering the sequence $\pi(t, j)$, obtained through the linear program $L B \_M 4$ '.

The vertex $J(t, j)$ has the following properties:

$\sum_{i=1}^{|I|} q_{i}(t, j)=t$

$0 \leq q_{i}(t, j) \leq d_{i}$

$$
i=1, \ldots,|I|
$$

$e_{k}^{c}(t, j)=\max \left\{(t+k-2) c+\hat{e}_{k, t},(t+k-1) c\right\} \quad k=1, \ldots,|K|$

Note that $\vec{e}^{c}(t, j)$ is the vector of corrected completion instants in accordance with the cycle time.

In short, a vertex $J(t, j)$ will be represented as follows:

$$
J(t, j)=\left\{(t, j), \vec{q}(t, j), \pi(t, j), L B(W(\pi(t, j))), \vec{e}(t, j), \vec{e}^{c}(t, j)\right\}
$$


At level 0 of the graph, there is only one $J(0)$ vertex. Initially, we may consider that at level $t, J(t)$ contains the vertices associated to all of the sub-sequences that can be built with $t$ products that satisfy properties (21), (22) and (23). However, it is easy to reduce the cardinal that $J(t)$ may present a priori, establishing the following definition of pseudo-dominance: given the sequences $\pi\left(t, j_{1}\right)$ and $\pi\left(t, j_{2}\right)$ associated to the vertices $J\left(t, j_{1}\right)$ and $J\left(t, j_{2}\right)$, then $\pi\left(t, j_{1}\right)$ pseudo-dominates $\pi\left(t, j_{2}\right)$ if:

$$
\pi\left(t, j_{1}\right) \prec \pi\left(t, j_{2}\right) \Leftrightarrow\left\{\begin{array}{l}
{\left[\vec{q}\left(t, j_{1}\right)=\vec{q}\left(t, j_{2}\right)\right] \text { and }} \\
{\left[L B\left(W\left(\pi\left(t, j_{1}\right)\right)\right) \leq L B\left(W\left(\pi\left(t, j_{2}\right)\right)\right)\right] \text { and }} \\
{\left[W\left(\pi\left(t, j_{1}\right)\right) \leq W\left(\pi\left(t, j_{2}\right)\right)\right] \text { and }} \\
{\left[\vec{e}^{c}\left(t, j_{1}\right) \leq \vec{e}^{c}\left(t, j_{2}\right)\right]}
\end{array}\right\}
$$

The reduction of $J(t)$ through the pseudo-dominances defined in (25) cannot guarantee the optimality of the solutions.

\section{The use of $B D P$}

For this study, we used a procedure based on $B D P$. This procedure combines features of dynamic programming (determination of extreme paths in graphs) with features of branch and bound algorithms. The principles of $B D P$ have been described by $[16,17]$. The procedure is described below (see details on [10]):

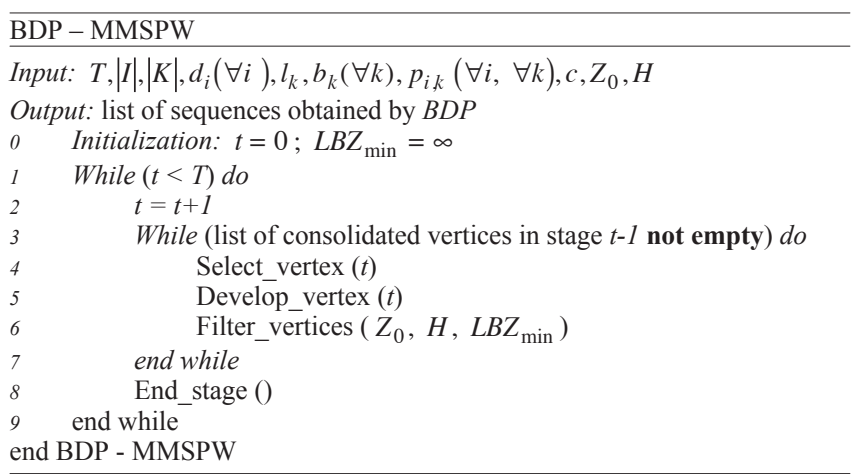

In the procedure appear the following functions:

- Select_vertex $(t)$ : selects, following a nondecreasing ordering of the $L B(W(\pi(t-1, j)))$ values, one of the vertices consolidated in stage $t-1$.

- Develop_vertex $(t)$ : develops the selected vertex in previous function adding a new product unit with pending demand.

- Filter_vertices $\left(Z_{0}, H, L B Z_{\text {min }}\right)$ : chooses, from all the vertices developed in the previous function, a maximum number $H$ of the most promising vertices (according to the lowest values of the lower bound $L B(W(\pi(t, j))))$, and removing those vertices in which their lower bound is greater than $Z_{0}$ (known initial solution)

- End_stage (): consolidates the most promising vertices in stage $t$ ( $H$ vertices as maximum).

\section{COMPUTATIONAL EXPERIMENT}

For the test operations of $M 4$ ' and the $B D P$ procedure, 225 instances from the literature were used. These instances were built from 45 production programs and 5 processing times structures, composed by four product types $(|I|=4)$ and four workstations $(|K|=4)$. These instances can be found in $[6,10]$.

The solutions for the 225 instances were obtained using the solver CPLEX v11.0 (single-processor license). Those solutions were compared with the solutions offered by the $B D P$ procedure proposed, under the following conditions and features:

1. $B D P$ procedure programmed in $\mathrm{C}++$, using gec v4.2.1, running on an Apple Macintosh iMac computer with an Intel Core i7 $2.93 \mathrm{GHz}$ processor and $8 \mathrm{~GB}$ RAM using MAC OS X 10.6.7 (not using any type of parallel code; therefore, the computer can be considered as a single $2.93 \mathrm{GHz}$ processor).

2. Six windows width $(H)$ were used, with values $1,4,16$, 64, 256 and 1024.

3. The initial solution $Z_{0}$ for each window width was the solution obtained by $B D P$ with the previous window width, except in the case $H=1$, where $Z_{0}$ was established as $\infty$.

4. To calculate the lower bounds, $L B(W(\pi(t, j)))$, of the overload associated to each vertex in the $B D P$ procedure, the solver Gurobi v4.5.0 was used, solving the linear program associated to $L B \_M 4$ '.

Tables II to VI show the results obtained by $B D P$ and $C P L E X$ for the 225 instances, grouped by processing times structures (1 to 5).

\section{TABLE II. RESULTS FOR STRUCTURE 1}

\begin{tabular}{|c|c|c|c|c|c|c|c|c|c|}
\hline \multirow{2}{*}{ Ins. } & \multirow{2}{*}{$\begin{array}{c}\text { Opt } \\
\text { CPLEX }\end{array}$} & $H=1$ & $H=4$ & $H=16$ & $H=64$ & $H=256$ & $H=1024$ & \multirow{2}{*}{$\begin{array}{c}\text { Best } \\
\text { found }\end{array}$} & \multirow{2}{*}{$\begin{array}{l}\text { Best } \\
R P D \\
\end{array}$} \\
\hline & & $W$ & $W$ & $W$ & $W$ & $W$ & $W$ & & \\
\hline$I / I$ & 40 & 46 & 42 & 40 & - & - & - & 40 & 0.00 \\
\hline $2 / 1$ & 100 & 107 & 102 & 100 & - & - & - & 100 & 0.00 \\
\hline $3 / 1$ & 81 & 88 & 86 & 81 & - & - & - & 81 & 0.00 \\
\hline $4 / 1$ & 143 & 143 & - & - & - & - & - & 143 & 0.00 \\
\hline $5 / 1$ & 46 & 49 & 49 & 46 & - & - & - & 46 & 0.00 \\
\hline $6 / 1$ & 46 & 49 & 47 & 47 & 46 & - & - & 46 & 0.00 \\
\hline $7 / 1$ & 14 & 17 & 16 & 14 & - & - & - & 14 & 0.00 \\
\hline $8 / 1$ & 61 & 63 & 63 & 61 & - & - & - & 61 & 0.00 \\
\hline $9 / 1$ & 81 & 87 & 81 & - & - & - & - & 81 & 0.00 \\
\hline $10 / 1$ & 68 & 76 & 69 & 68 & - & - & - & 68 & 0.00 \\
\hline $11 / 1$ & 37 & 48 & 43 & 40 & 39 & 37 & - & 37 & 0.00 \\
\hline $12 / 1$ & 37 & 48 & 44 & 38 & 37 & - & - & 37 & 0.00 \\
\hline $13 / 1$ & 29 & 38 & 34 & 30 & 30 & 29 & - & 29 & 0.00 \\
\hline $14 / 1$ & 45 & 50 & 48 & 47 & 46 & 45 & - & 45 & 0.00 \\
\hline $15 / 1$ & 40 & 51 & 42 & 41 & 41 & 40 & - & 40 & 0.00 \\
\hline $16 / 1$ & 32 & 40 & 36 & 34 & 34 & 32 & - & 32 & 0.00 \\
\hline $17 / 1$ & 37 & 48 & 42 & 39 & 38 & 37 & - & 37 & 0.00 \\
\hline $18 / 1$ & 45 & 55 & 53 & 47 & 46 & 45 & - & 45 & 0.00 \\
\hline $19 / 1$ & 33 & 38 & 35 & 35 & 33 & - & - & 33 & 0.00 \\
\hline $20 / 1$ & 31 & 38 & 31 & - & - & - & - & 31 & 0.00 \\
\hline $21 / 1$ & 56 & 72 & 57 & 57 & 56 & - & - & 56 & 0.00 \\
\hline $22 / 1$ & 63 & 96 & 79 & 63 & - & - & - & 63 & 0.00 \\
\hline $23 / 1$ & 55 & 72 & 64 & 60 & 55 & - & - & 55 & 0.00 \\
\hline $24 / 1$ & 67 & 90 & 78 & 67 & - & - & - & 67 & 0.00 \\
\hline $25 / 1$ & 56 & 67 & 60 & 58 & 56 & - & - & 56 & 0.00 \\
\hline $26 / 1$ & 63 & 74 & 69 & 63 & 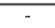 & - & - & 63 & 0.00 \\
\hline $27 / 1$ & 58 & 63 & 63 & 59 & 58 & - & - & 58 & 0.00 \\
\hline $28 / 1$ & 46 & 57 & 52 & 46 & - & - & - & 46 & 0.00 \\
\hline $29 / 1$ & 48 & 57 & 53 & 50 & 48 & - & - & 48 & 0.00 \\
\hline $30 / 1$ & 55 & 68 & 55 & - & - & - & - & 55 & 0.00 \\
\hline $31 / 1$ & 52 & 58 & 55 & 53 & 52 & - & - & 52 & 0.00 \\
\hline $32 / 1$ & 53 & 62 & 53 & - & - & - & - & 53 & 0.00 \\
\hline $33 / 1$ & 53 & 63 & 57 & 53 & - & - & - & 53 & 0.00 \\
\hline $34 / 1$ & 25 & 35 & 30 & 25 & - & - & - & 25 & 0.00 \\
\hline $35 / 1$ & 41 & 46 & 42 & 41 & - & - & - & 41 & 0.00 \\
\hline $36 / 1$ & 29 & 37 & 35 & 29 & - & - & - & 29 & 0.00 \\
\hline $37 / 1$ & 48 & 58 & 52 & 49 & 48 & - & - & 48 & 0.00 \\
\hline $38 / 1$ & 43 & 50 & 45 & 45 & 43 & - & - & 43 & 0.00 \\
\hline $39 / 1$ & 47 & 58 & 49 & 48 & 47 & - & - & 47 & 0.00 \\
\hline $40 / 1$ & 22 & 30 & 23 & 23 & 22 & - & - & 22 & 0.00 \\
\hline $41 / 1$ & 34 & 39 & 35 & 35 & 34 & - & - & 34 & 0.00 \\
\hline $42 / 1$ & 22 & 30 & 24 & 24 & 23 & 22 & - & 22 & 0.00 \\
\hline $43 / 1$ & 44 & 52 & 49 & 45 & 45 & 44 & - & 44 & 0.00 \\
\hline $44 / 1$ & 34 & 39 & 37 & 35 & 34 & - & - & 34 & 0.00 \\
\hline $45 / 1$ & 43 & 55 & 49 & 45 & 44 & 43 & - & 43 & 0.00 \\
\hline
\end{tabular}


TABLE III. RESULTS FOR STRUCTURE 2

\begin{tabular}{|c|c|c|c|c|c|c|c|c|c|}
\hline \multirow{2}{*}{ Ins. } & \multirow{2}{*}{$\begin{array}{c}\text { Opt } \\
\text { CPLEX }\end{array}$} & $H=1$ & $H=4$ & $H=16$ & $H=64$ & $H=256$ & $H=1024$ & \multirow{2}{*}{$\begin{array}{c}\text { Best } \\
\text { found }\end{array}$} & \multirow{2}{*}{$\begin{array}{l}\text { Best } \\
\text { RPD }\end{array}$} \\
\hline & & $W$ & $W$ & $W$ & $W$ & $W$ & $W$ & & \\
\hline $1 / 2$ & 230 & 230 & - & - & - & - & - & 230 & 0.00 \\
\hline $2 / 2$ & 282 & 296 & 296 & 282 & - & - & - & 282 & 0.00 \\
\hline $3 / 2$ & 270 & 271 & 271 & 270 & - & - & - & 270 & 0.00 \\
\hline $4 / 2$ & 324 & 328 & 324 & - & - & - & - & 324 & 0.00 \\
\hline $5 / 2$ & 200 & 227 & 203 & 200 & - & - & - & 200 & 0.00 \\
\hline $6 / 2$ & 100 & 114 & 108 & 100 & & - & - & 100 & 0.00 \\
\hline $7 / 2$ & 151 & 160 & 155 & 152 & 151 & - & - & 151 & 0.00 \\
\hline $8 / 2$ & 199 & 218 & 205 & 203 & 201 & 199 & - & 199 & 0.00 \\
\hline $9 / 2$ & 218 & 238 & 224 & 218 & - & - & - & 218 & 0.00 \\
\hline $10 / 2$ & 225 & 234 & 225 & - & - & - & - & 225 & 0.00 \\
\hline $11 / 2$ & 132 & 162 & 139 & 136 & 132 & - & - & 132 & 0.00 \\
\hline $12 / 2$ & 112 & 129 & 123 & 113 & 112 & - & - & 112 & 0.00 \\
\hline $13 / 2$ & 122 & 145 & 122 & - & - & - & - & 122 & 0.00 \\
\hline $14 / 2$ & 153 & 169 & 163 & 153 & - & - & - & 153 & 0.00 \\
\hline $15 / 2$ & 147 & 161 & 153 & 147 & - & - & - & 147 & 0.00 \\
\hline $16 / 2$ & 117 & 132 & 123 & 117 & - & - & - & 117 & 0.00 \\
\hline $17 / 2$ & 133 & 151 & 140 & 133 & - & - & - & 133 & 0.00 \\
\hline $18 / 2$ & 136 & 160 & 138 & 138 & 138 & 137 & 136 & 136 & 0.00 \\
\hline $19 / 2$ & 150 & 179 & 162 & 150 & - & - & - & 150 & 0.00 \\
\hline $20 / 2$ & 114 & 131 & 119 & 115 & 114 & - & - & 114 & 0.00 \\
\hline $21 / 2$ & 193 & 196 & 195 & 193 & 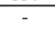 & - & - & 193 & 0.00 \\
\hline $22 / 2$ & 213 & 227 & 218 & 213 & - & - & - & 213 & 0.00 \\
\hline $23 / 2$ & 197 & 213 & 203 & 197 & - & - & - & 197 & 0.00 \\
\hline $24 / 2$ & 205 & 210 & 210 & 208 & 208 & 205 & - & 205 & 0.00 \\
\hline $25 / 2$ & 190 & 196 & 196 & 190 & - & - & - & 190 & 0.00 \\
\hline $26 / 2$ & 206 & 214 & 214 & 208 & 206 & - & - & 206 & 0.00 \\
\hline $27 / 2$ & 200 & 211 & 202 & 201 & 200 & - & - & 200 & 0.00 \\
\hline $28 / 2$ & 175 & 189 & 189 & 175 & - & - & - & 175 & 0.00 \\
\hline $29 / 2$ & 158 & 194 & 178 & 158 & - & - & - & 158 & 0.00 \\
\hline $30 / 2$ & 178 & 186 & 183 & 179 & 179 & 178 & - & 178 & 0.00 \\
\hline $31 / 2$ & 153 & 184 & 159 & 159 & 155 & 154 & 153 & 153 & 0.00 \\
\hline $32 / 2$ & 187 & 204 & 202 & 188 & 188 & 187 & - & 187 & 0.00 \\
\hline $33 / 2$ & 174 & 194 & 177 & 174 & - & - & - & 174 & 0.00 \\
\hline $34 / 2$ & 141 & 155 & 146 & 141 & - & - & - & 141 & 0.00 \\
\hline $35 / 2$ & 117 & 145 & 126 & 121 & 117 & - & - & 117 & 0.00 \\
\hline $36 / 2$ & 152 & 156 & 152 & - & 7 & - & - & 152 & 0.00 \\
\hline $37 / 2$ & 116 & 144 & 124 & 122 & 116 & - & - & 116 & 0.00 \\
\hline $38 / 2$ & 179 & 209 & 185 & 179 & - & - & - & 179 & 0.00 \\
\hline $39 / 2$ & 168 & 190 & 168 & - & - & - & - & 168 & 0.00 \\
\hline $40 / 2$ & 104 & 119 & 111 & 104 & - & - & - & 104 & 0.00 \\
\hline $41 / 2$ & 92 & 106 & 101 & 92 & - & - & - & 92 & 0.00 \\
\hline $42 / 2$ & 137 & 157 & 148 & 140 & 137 & - & - & 137 & 0.00 \\
\hline $43 / 2$ & 116 & 135 & 120 & 116 & - & - & - & 116 & 0.00 \\
\hline $44 / 2$ & 163 & 185 & 163 & - & - & - & - & 163 & 0.00 \\
\hline $45 / 2$ & 149 & 161 & 149 & - & - & - & - & 149 & 0.00 \\
\hline
\end{tabular}

TABLE IV. RESULTS FOR STRUCTURE 3

\begin{tabular}{|c|c|c|c|c|c|c|c|c|c|}
\hline \multirow{2}{*}{ Ins. } & \multirow{2}{*}{$\begin{array}{c}\text { Opt } \\
\text { CPLEX }\end{array}$} & $H=1$ & $H=4$ & $H=16$ & $H=64$ & $H=256$ & $H=1024$ & \multirow{2}{*}{$\begin{array}{l}\text { Best } \\
\text { found }\end{array}$} & \multirow{2}{*}{$\begin{array}{l}\text { Best } \\
R P D \\
\end{array}$} \\
\hline & & $W$ & $W$ & $W$ & $W$ & $W$ & $W$ & & \\
\hline $1 / 3$ & 369 & 399 & 386 & 369 & - & - & - & 369 & 0.00 \\
\hline $2 / 3$ & 339 & 373 & 356 & 341 & 339 & - & - & 339 & 0.00 \\
\hline $3 / 3$ & 421 & 444 & 434 & 421 & - & - & - & 421 & 0.00 \\
\hline $4 / 3$ & 313 & 329 & 321 & 314 & 313 & - & - & 313 & 0.00 \\
\hline $5 / 3$ & 345 & 397 & 364 & 349 & 345 & - & - & 345 & 0.00 \\
\hline $6 / 3$ & 227 & 244 & 227 & 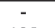 & - & - & - & 227 & 0.00 \\
\hline $7 / 3$ & 185 & 211 & 199 & 185 & - & - & - & 185 & 0.00 \\
\hline $8 / 3$ & 279 & 290 & 285 & 279 & - & - & - & 279 & 0.00 \\
\hline $9 / 3$ & 232 & 243 & 241 & 238 & 232 & - & - & 232 & 0.00 \\
\hline $10 / 3$ & 367 & 383 & 381 & 373 & 367 & - & - & 367 & 0.00 \\
\hline $11 / 3$ & 215 & 276 & 246 & 231 & 224 & 215 & - & 215 & 0.00 \\
\hline $12 / 3$ & 221 & 243 & 234 & 223 & 221 & - & - & 221 & 0.00 \\
\hline $13 / 3$ & 207 & 240 & 221 & 212 & 210 & 207 & - & 207 & 0.00 \\
\hline $14 / 3$ & 231 & 260 & 252 & 244 & 240 & 237 & 237 & 237 & 2.60 \\
\hline $15 / 3$ & 239 & 285 & 268 & 267 & 249 & 239 & - & 239 & 0.00 \\
\hline $16 / 3$ & 196 & 222 & 210 & 210 & 200 & 196 & - & 196 & 0.00 \\
\hline $17 / 3$ & 219 & 236 & 236 & 229 & 225 & 221 & 221 & 221 & 0.91 \\
\hline $18 / 3$ & 229 & 262 & 242 & 238 & 229 & - & - & 229 & 0.00 \\
\hline $19 / 3$ & 217 & 284 & 259 & 226 & 219 & 217 & - & 217 & 0.00 \\
\hline $20 / 3$ & 221 & 253 & 245 & 236 & 221 & - & - & 221 & 0.00 \\
\hline $21 / 3$ & 277 & 303 & 303 & 290 & 281 & 277 & - & 277 & 0.00 \\
\hline $22 / 3$ & 311 & 336 & 332 & 325 & 314 & 311 & - & 311 & 0.00 \\
\hline $23 / 3$ & 329 & 354 & 350 & 343 & 330 & 329 & - & 329 & 0.00 \\
\hline $24 / 3$ & 259 & 284 & 276 & 269 & 263 & 259 & - & 259 & 0.00 \\
\hline $25 / 3$ & 295 & 321 & 314 & 307 & 299 & 295 & - & 295 & 0.00 \\
\hline $26 / 3$ & 243 & 263 & 256 & 248 & 245 & 245 & 245 & 245 & 0.82 \\
\hline $27 / 3$ & 261 & 272 & 272 & 261 & - & - & - & 261 & 0.00 \\
\hline $28 / 3$ & 265 & 309 & 296 & 296 & 273 & 265 & - & 265 & 0.00 \\
\hline $29 / 3$ & 283 & 327 & 321 & 310 & 299 & 283 & - & 283 & 0.00 \\
\hline $30 / 3$ & 214 & 239 & 225 & 217 & 214 & - & - & 214 & 0.00 \\
\hline $31 / 3$ & 245 & 267 & 267 & 257 & 247 & 247 & 247 & 247 & 0.82 \\
\hline $32 / 3$ & 235 & 281 & 259 & 248 & 235 & - & - & 235 & 0.00 \\
\hline $33 / 3$ & 243 & 273 & 258 & 243 & - & - & - & 243 & 0.00 \\
\hline $34 / 3$ & 208 & 235 & 222 & 208 & - & - & - & 208 & 0.00 \\
\hline $35 / 3$ & 235 & 266 & 257 & 251 & 235 & - & - & 235 & 0.00 \\
\hline $36 / 3$ & 196 & 229 & 215 & 199 & 196 & 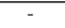 & - & 196 & 0.00 \\
\hline $37 / 3$ & 235 & 253 & 241 & 237 & 235 & - & - & 235 & 0.00 \\
\hline $38 / 3$ & 263 & 333 & 306 & 287 & 265 & 263 & - & 263 & 0.00 \\
\hline $39 / 3$ & 257 & 326 & 294 & 274 & 259 & 257 & - & 257 & 0.00 \\
\hline $40 / 3$ & 199 & 222 & 213 & 199 & - & - & - & 199 & 0.00 \\
\hline $41 / 3$ & 213 & 230 & 217 & 213 & - & - & - & 213 & 0.00 \\
\hline $42 / 3$ & 211 & 292 & 242 & 233 & 211 & - & - & 211 & 0.00 \\
\hline $43 / 3$ & 232 & 257 & 248 & 235 & 232 & - & - & 232 & 0.00 \\
\hline $44 / 3$ & 271 & 341 & 314 & 281 & 271 & - & - & 271 & 0.00 \\
\hline $45 / 3$ & 265 & 334 & 306 & 285 & 270 & 265 & - & 265 & 0.00 \\
\hline
\end{tabular}

TABLE V. RESULTS FOR STRUCTURE 4

\begin{tabular}{|c|c|c|c|c|c|c|c|c|c|}
\hline \multirow{2}{*}{ Ins. } & \multirow{2}{*}{$\begin{array}{c}\text { Opt } \\
\text { CPLEX }\end{array}$} & $H=1$ & $H=4$ & $H=16$ & $H=64$ & $H=256$ & $H=1024$ & \multirow{2}{*}{$\begin{array}{l}\text { Best } \\
\text { found }\end{array}$} & \multirow{2}{*}{$\begin{array}{l}\text { Best } \\
R P D\end{array}$} \\
\hline & & $W$ & $W$ & $W$ & $W$ & $W$ & $W$ & & \\
\hline $1 / 4$ & 710 & 771 & 771 & 730 & 710 & - & - & 710 & 0.00 \\
\hline $2 / 4$ & 638 & 699 & 681 & 662 & 638 & - & - & 638 & 0.00 \\
\hline $3 / 4$ & 80 & 80 & - & - & - & - & - & 80 & 0.00 \\
\hline $4 / 4$ & 80 & 80 & - & - & - & - & - & 80 & 0.00 \\
\hline $5 / 4$ & 674 & 735 & 735 & 735 & 682 & 682 & 682 & 682 & 1.19 \\
\hline $6 / 4$ & 338 & 338 & - & - & - & - & - & 338 & 0.00 \\
\hline $7 / 4$ & 338 & 338 & - & - & - & - & - & 338 & 0.00 \\
\hline $8 / 4$ & 302 & 307 & 302 & - & - & - & - & 302 & 0.00 \\
\hline $9 / 4$ & 302 & 302 & - & - & - & - & - & 302 & 0.00 \\
\hline $10 / 4$ & 80 & 80 & - & - & - & - & - & 80 & 0.00 \\
\hline $11 / 4$ & 415 & 430 & 425 & 416 & 415 & - & - & 415 & 0.00 \\
\hline $12 / 4$ & 326 & 326 & - & - & - & - & - & 326 & 0.00 \\
\hline $13 / 4$ & 326 & 326 & - & - & - & - & - & 326 & 0.00 \\
\hline $14 / 4$ & 314 & 319 & 314 & - & - & - & - & 314 & 0.00 \\
\hline $15 / 4$ & 240 & 240 & - & - & - & - & - & 240 & 0.00 \\
\hline $16 / 4$ & 240 & 240 & - & - & - & - & - & 240 & 0.00 \\
\hline $17 / 4$ & 320 & 320 & - & - & - & - & - & 320 & 0.00 \\
\hline $18 / 4$ & 415 & 415 & - & - & - & - & - & 415 & 0.00 \\
\hline $19 / 4$ & 415 & 425 & 425 & 417 & 415 & - & - & 415 & 0.00 \\
\hline $20 / 4$ & 252 & 252 & - & - & - & - & - & 252 & 0.00 \\
\hline $21 / 4$ & 228 & 233 & 228 & - & - & - & - & 228 & 0.00 \\
\hline $22 / 4$ & 154 & 154 & - & - & - & - & - & 154 & 0.00 \\
\hline $23 / 4$ & 154 & 154 & - & - & - & - & - & 154 & 0.00 \\
\hline $24 / 4$ & 228 & 228 & - & - & - & - & - & 228 & 0.00 \\
\hline $25 / 4$ & 228 & 233 & 228 & - & - & - & - & 228 & 0.00 \\
\hline $26 / 4$ & 302 & 307 & 302 & - & - & - & - & 302 & 0.00 \\
\hline $27 / 4$ & 302 & 307 & 302 & - & - & - & - & 302 & 0.00 \\
\hline $28 / 4$ & 166 & 166 & - & - & - & - & - & 166 & 0.00 \\
\hline $29 / 4$ & 166 & 166 & - & - & - & - & - & 166 & 0.00 \\
\hline $30 / 4$ & 314 & 314 & - & - & - & - & - & 314 & 0.00 \\
\hline $31 / 4$ & 314 & 319 & 314 & - & - & - & - & 314 & 0.00 \\
\hline $32 / 4$ & 403 & 413 & 413 & 403 & - & - & - & 403 & 0.00 \\
\hline $33 / 4$ & 403 & 403 & - & - & - & - & - & 403 & 0.00 \\
\hline $34 / 4$ & 252 & 252 & - & - & - & - & - & 252 & 0.00 \\
\hline $35 / 4$ & 252 & 252 & - & - & - & - & - & 252 & 0.00 \\
\hline $36 / 4$ & 326 & 326 & - & - & - & - & - & 326 & 0.00 \\
\hline $37 / 4$ & 326 & 326 & - & - & - & - & - & 326 & 0.00 \\
\hline $38 / 4$ & 510 & 564 & 564 & 564 & 541 & 516 & 516 & 516 & 1.18 \\
\hline $39 / 4$ & 509 & 564 & 564 & 554 & 524 & 511 & 511 & 511 & 0.39 \\
\hline $40 / 4$ & 338 & 338 & - & - & - & - & - & 338 & 0.00 \\
\hline $41 / 4$ & 338 & 338 & - & - & - & - & - & 338 & 0.00 \\
\hline $42 / 4$ & 427 & 433 & 433 & 433 & 427 & - & - & 427 & 0.00 \\
\hline $43 / 4$ & 427 & 429 & 428 & 428 & 427 & - & - & 427 & 0.00 \\
\hline $44 / 4$ & 522 & 576 & 576 & 576 & 538 & 531 & 531 & 531 & 1.72 \\
\hline $45 / 4$ & 521 & 576 & 566 & 566 & 542 & 536 & 521 & 521 & 0.00 \\
\hline
\end{tabular}

TABLE VI. RESULTS FOR STRUCTURE 5

\begin{tabular}{|c|c|c|c|c|c|c|c|c|c|}
\hline & Opt & $H=1$ & $H=4$ & $H=16$ & $H=64$ & $H=256$ & $H=1024$ & Best & Best \\
\hline Ins. & CPLEX & $W$ & $W$ & $W$ & $W$ & $W$ & $W$ & found & $R P D$ \\
\hline $1 / 5$ & 211 & 228 & 221 & 211 & - & - & - & 211 & 0.00 \\
\hline $2 / 5$ & 276 & 285 & 285 & 276 & - & - & - & 276 & 0.00 \\
\hline $3 / 5$ & 166 & 167 & 166 & - & - & - & - & 166 & 0.00 \\
\hline $4 / 5$ & 212 & 232 & 212 & - & - & - & - & 212 & 0.00 \\
\hline $5 / 5$ & 222 & 237 & 227 & 227 & 227 & 223 & 222 & 222 & 0.00 \\
\hline $6 / 5$ & 136 & 144 & 137 & 136 & - & - & - & 136 & 0.00 \\
\hline $7 / 5$ & 143 & 163 & 145 & 143 & - & - & - & 143 & 0.00 \\
\hline $8 / 5$ & 177 & 223 & 178 & 178 & 177 & - & - & 177 & 0.00 \\
\hline $9 / 5$ & 175 & 196 & 177 & 177 & 176 & 176 & 176 & 176 & 0.57 \\
\hline $10 / 5$ & 42 & 47 & 42 & - & - & - & - & 42 & 0.00 \\
\hline $11 / 5$ & 121 & 136 & 131 & 121 & - & - & - & 121 & 0.00 \\
\hline $12 / 5$ & 95 & 112 & 95 & - & - & - & - & 95 & 0.00 \\
\hline $13 / 5$ & 95 & 107 & 96 & 96 & 95 & - & - & 95 & 0.00 \\
\hline $14 / 5$ & 103 & 114 & 108 & 103 & - & - & - & 103 & 0.00 \\
\hline $15 / 5$ & 70 & 75 & 70 & - & - & - & - & 70 & 0.00 \\
\hline $16 / 5$ & 65 & 70 & 65 & - & - & - & - & 65 & 0.00 \\
\hline $17 / 5$ & 84 & 111 & 89 & 89 & 84 & - & - & 84 & 0.00 \\
\hline $18 / 5$ & 158 & 166 & 166 & 159 & 159 & 158 & - & 158 & 0.00 \\
\hline $19 / 5$ & 151 & 186 & 154 & 151 & - & - & - & 151 & 0.00 \\
\hline $20 / 5$ & 68 & 96 & 79 & 68 & - & - & - & 68 & 0.00 \\
\hline $21 / 5$ & 106 & 108 & 107 & 106 & - & - & - & 106 & 0.00 \\
\hline $22 / 5$ & 82 & 104 & 83 & 83 & 82 & - & - & 82 & 0.00 \\
\hline $23 / 5$ & 67 & 93 & 77 & 67 & - & - & - & 67 & 0.00 \\
\hline $24 / 5$ & 128 & 145 & 129 & 129 & 128 & - & - & 128 & 0.00 \\
\hline $25 / 5$ & 103 & 132 & 114 & 103 & 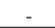 & - & - & 103 & 0.00 \\
\hline $26 / 5$ & 152 & 165 & 154 & 154 & 153 & 152 & - & 152 & 0.00 \\
\hline $27 / 5$ & 137 & 150 & 141 & 141 & 137 & - & - & 137 & 0.00 \\
\hline $28 / 5$ & 64 & 89 & 65 & 64 & - & - & - & 64 & 0.00 \\
\hline $29 / 5$ & 56 & 70 & 56 & - & - & - & - & 56 & 0.00 \\
\hline $30 / 5$ & 155 & 168 & 156 & 155 & - & - & - & 155 & 0.00 \\
\hline $31 / 5$ & 148 & 162 & 155 & 149 & 148 & - & - & 148 & 0.00 \\
\hline $32 / 5$ & 176 & 196 & 181 & 177 & 176 & - & - & 176 & 0.00 \\
\hline $33 / 5$ & 171 & 176 & 176 & 172 & 172 & 171 & - & 171 & 0.00 \\
\hline $34 / 5$ & 91 & 128 & 92 & 91 & - & - & - & 91 & 0.00 \\
\hline $35 / 5$ & 86 & 121 & 92 & 86 & - & - & - & 86 & 0.00 \\
\hline $36 / 5$ & 146 & 163 & 146 & - & - & - & - & 146 & 0.00 \\
\hline $37 / 5$ & 132 & 132 & - & . & - & - & - & 132 & 0.00 \\
\hline $38 / 5$ & 185 & 206 & 188 & 188 & 188 & 186 & 185 & 185 & 0.00 \\
\hline $39 / 5$ & 194 & 205 & 198 & 196 & 196 & 196 & 196 & 196 & 1.03 \\
\hline $40 / 5$ & 103 & 119 & 104 & 103 & - & - & - & 103 & 0.00 \\
\hline $41 / 5$ & 107 & 130 & 117 & 107 & 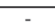 & - & - & 107 & 0.00 \\
\hline $42 / 5$ & 142 & 155 & 145 & 142 & - & - & - & 142 & 0.00 \\
\hline $43 / 5$ & 151 & 152 & 152 & 152 & 152 & 152 & 152 & 152 & 0.66 \\
\hline $44 / 5$ & 169 & 182 & 171 & 169 & - & - & - & 169 & 0.00 \\
\hline $45 / 5$ & 182 & 189 & 187 & 183 & 183 & 183 & 183 & 183 & 0.55 \\
\hline
\end{tabular}


For each structure (Tables II to VI) are shown: (1) the code of the instance (production program/structure) in the column "Ins."; (2) the value of the total overload $W$ of the optimal solution for each instance obtained by CPLEX ("Opt $C P L E X$ "); (3) the values of the overloads $W$ obtained by the $B D P$ algorithm using different windows width $H$ $(H=1, \ldots, 1024)$; (4) the best value of $W$, for each instance, found by BDP ("Best found"); and (5) the best value, for each instance, of the relative percentage deviation $(R P D)$ obtained as $R P D=(($ Best found - Opt CPLEX $) /$ Opt CPLEX $) * 100$ (“Best RPD”).

The $B D P$ procedure, with the incorporation of the pseudo-dominances defined in (25), reaches the optimal result for 213 of the 225 instances. The instances in which the optimum was not reached are focused in structure 3 (production programs 14, 17, 26 and 31), structure 4 (production programs 5, 38, 39 and 44) and structure 5 (production programs 9, 39, 43 and 45) are shown in Tables IV, $\mathrm{V}$ and VI, respectively. The average values of the $R P D$ for the instances of these structures are $0.11 \%, 0.10 \%$ and $0.06 \%$.

In order to compare the computational efficiency, in CPU times, of $B D P$ against the exact procedure in solver CPLEX, we have summarized the CPU times taken to obtain the best solutions of the 225 instances. Table VII show the minimum, maximum, and average CPU times, employed by both procedures to solve the set of instances grouped by production programs and processing times structures.

TABLE VII. CPU TIMES BY BLOCKS AND STRUCTURES

\begin{tabular}{|c|ccc|ccc|}
\cline { 2 - 7 } \multicolumn{1}{c|}{} & \multicolumn{3}{c|}{$M 4^{\prime}-C P L E X$} & \multicolumn{3}{c|}{ BDP } \\
\cline { 2 - 7 } \multicolumn{1}{c|}{} & CPU min & CPU max & Average & CPU min & CPU max & Average \\
\hline Block 1 & 0.0 & 1.5 & 0.4 & 0.0 & 1.3 & 0.5 \\
Block 2 & 0.0 & 173.9 & 23.3 & 0.0 & 9.5 & 1.7 \\
Block 3 & 5.1 & 2533.2 & 335.7 & 0.1 & 22.6 & 6.4 \\
Block 4 & 6.6 & 111.5 & 35.2 & 0.1 & 10.5 & 3.7 \\
Block 5 & 0.1 & 697.7 & 43.5 & 0.1 & 14.2 & 3.2 \\
\hline Structure 1 & 0.0 & 301.3 & 46.9 & 0.0 & 16.6 & 4.3 \\
Structure 2 & 0.1 & 129.7 & 16.5 & 0.1 & 7.2 & 2.3 \\
Structure 3 & 0.1 & 180.3 & 32.2 & 0.3 & 22.6 & 5.9 \\
Structure 4 & 0.0 & 2533.2 & 288.5 & 0.0 & 7.2 & 1.2 \\
Structure 5 & 0.2 & 126.4 & 24.4 & 0.1 & 14.2 & 2.8 \\
\hline
\end{tabular}

In all cases (except Block 1), the average CPU times used by CPLEX are larger than the corresponding to the $B D P$, being the Structure 4 the extreme case, where the $B D P$ was 239 times faster than CPLEX, and on average 54 times faster.

\section{CONCLUSIONS}

We have proposed a heuristic procedure based on $B D P$ to solve a variant of the $M M S P-W$, specifically the variant with serial workstations and unrestricted interruption of the operations.

The proposed procedure was computationally competitive against CPLEX. The $B D P$ procedure obtained 213 of 225 optimums in the instances used; in the remaining 12 instances, $B D P$ obtained solutions, on average, far less than $0.1 \%$ over the optimal value. The CPU times shows that $B D P$ was 54 times faster than CPLEX.

In future research, our work will be focused on exploiting and improving the procedure in an industrial environment.

\section{ACKNOWLEDGMENT}

The authors greatly appreciate the collaboration of Nissan Spanish Industrial Operations (NSIO). This work was funded by Nissan Chair UPC and project PROTHIUS-III, DPI2010-16759, including EDRF funding from the Spanish government.

\section{REFERENCES}

[1] N. Boysen, M. Fliedner and A. Scholl, "Sequencing mixed-model assembly lines: survey, classification and model critique", European Journal of Operational Research, 192/2, 349-373, 2009.

[2] C. A. Yano and R. Rachamadugu, "Sequencing to minimize work overload in assembly lines with product options", Management Science, 37/5, 572-586, 1991.

[3] J. Bautista, J. Pereira and B. Adenso-Díaz, "A GRASP approach for the extended car sequencing problem", Journal of Scheduling, 11/1, 3-16, 2008

[4] K. Okamura and H. Yamashina, "A heuristic algorithm for the assembly line model-mix sequencing problem to minimize the risk of stopping the conveyor", International Journal of Production Research, 17/3, 233-247, 1979.

[5] Z. Xiaobo and K. Ohno, "Algorithms for sequencing mixed models on assembly line in a JIT production system", Computers \& Industrial Engineering, 31/1, 47-56, 1997.

[6] J. Bautista and J. Cano, "Minimizing work overload in mixed-model assembly lines", International Journal of Production Economics, 112/1, 177-191, 2008.

[7] A. Bolat, "Efficient methods for sequencing minimum job sets on mixed model assembly lines", Naval Research Logistics, 44/5, 419437, 1997.

[8] L.H. Tsai, "Mixed-model sequencing to minimize utility work and the risk of conveyor stoppage", Management Science, 41/3, 485-495, 1995.

[9] A. Bolat, "A mathematical model for sequencing mixed models with due dates", International Journal of Production Research, 41/5, 897918, 2003.

[10] J. Bautista and A. Cano, "Solving mixed model sequencing problem in assembly lines with serial workstations with work overload minimisation and interruption rules", European Journal of Operational Research, 210/3, 495-513, 2011.

[11] C. A. Yano and A. Bolat, "Survey, development, and application of algorithms for sequencing paced assembly lines", Journal of Manufacturing and Operations Management, 2/3, 172-198, 1989.

[12] A. Bolat and C.A. Yano, "Scheduling algorithms to minimize utility work at a single station on paced assembly line", Production Planning and Control, 3/4, 393-405, 1992.

[13] A. Scholl, R. Klein and W. Domschke, "Pattern based vocabulary building for effectively sequencing mixed-model assembly lines", Journal of Heuristics, 4/4, 359-381, 1998.

[14] J. Cano, R. Ríos and J. Bautista, "A scatter search based hyperheuristic for sequencing a mixed-model assembly line", Journal of Heuristics, 6/6, 749-770, 2010.

[15] E. Erel, Y. Gocgun and I. Sabuncuoglu, "Mixed-model assembly line sequencing using beam search", International Journal of Production Research, 45/22, 5265-5284, 2007.

[16] J. Bautista, "Procedimientos heurísticos y exactos para la secuenciación en sistemas productivos de unidades homogéneas (contexto J.I.T.)". Doctoral Thesis, DOE, ETSEIB-UPC, 1993.

[17] J. Bautista, R. Companys and A. Corominas, "Heuristics and exact algorithms for solving the Monden problem", European Journal of Operational Research, 88/1, 101-113, 1996.

[18] J. Bautista and J. Pereira, "Procedures for the time and space constrained assembly line balancing problem", European Journal of Operational Research, 212/3, 473-481, 2011. 